\title{
Speed Control Based on ESO for the Pitching Axis of Satellite Cameras
}

\author{
BingYou Liu, ${ }^{1,2}$ Yi Jin, ${ }^{1}$ ChangZheng Chen, ${ }^{3}$ and HuiCheng Yang ${ }^{2}$ \\ ${ }^{1}$ Department of Precision Machinery and Precision Instrumentation, University of Science and Technology of China, \\ Hefei 230027, China \\ ${ }^{2}$ Key Lab of Electric and Control of Anhui Province, Anhui Polytechnic University, Wuhu 241000, China \\ ${ }^{3}$ Changchun Institute of Optics, Fine Mechanics and Physics, Chinese Academy of Sciences, Changchun 130033, China
}

Correspondence should be addressed to Yi Jin; jinyi08@ustc.edu.cn

Received 20 November 2015; Revised 19 February 2016; Accepted 13 March 2016

Academic Editor: Mingcong Deng

Copyright (c) 2016 BingYou Liu et al. This is an open access article distributed under the Creative Commons Attribution License, which permits unrestricted use, distribution, and reproduction in any medium, provided the original work is properly cited.

\begin{abstract}
The pitching axis is the main axis of a satellite camera and is used to control the pitch posture of satellite cameras. A control strategy based on extended state observer (ESO) is designed to obtain a fast response speed and highly accurate pitching axis control system and eliminate disturbances during the adjustment of pitch posture. First, a sufficient condition of stabilization for ESO is obtained by analyzing the steady-state error of the system under step input. Parameter tuning and disturbance compensation are performed by ESO. Second, the ESO of speed loop is designed by the speed equation of the pitching axis of satellite cameras. The ESO parameters are obtained by pole assignment. By ESO, the original state variable observes the motor angular speed and the extended state variable observes the load torque. Therefore, the external load disturbances of the control system are estimated in real time. Finally, simulation experiments are performed for the system on the cases of nonload starting, adding external disturbances on the system suddenly, and the load of system changing suddenly. Simulation results show that the control strategy based on ESO has better stability, adaptability, and robustness than the PI control strategy.
\end{abstract}

\section{Introduction}

Pitching axis is an important axis of the posture control system of a satellite camera. The control system of pitching axis adopts a three-loop servo control system: the current loop, speed loop, and position loop. The disturbances of the speed loop are greater than those in the other two loops. Therefore, the speed loop should be controlled in real time to achieve good performances for the control system of the pitching axis. This paper uses an extended state observer (ESO) in the speed loop control system to obtain good performances.

A control strategy based on ESO was first presented in [1]. The main idea of the control strategy is to extend the disturbance that influences the output of the controlled object into a new state and establish an observer to observe this state by using a special feedback method. The control strategy is a good control method for solving the problem of uncertain nonlinear system. The control strategy has various features, such as high-control accuracy, fast response speed, and good robust performance, and does not rely on the model of a controlled object. Therefore, this control strategy is widely used in the field of aeronautics and astronautics, precision machinery, modern weapon systems, and so on.

Extensive research on the application of ESO has been conducted locally and internationally and has shown some achievements. For example, Yang and Huang [2] analyzed the capability of ESO in estimating uncertainty. Wang et al. [3] proved the second-order nonlinear stability of ESO by the Lyapunov function. Shao et al. [4] not only extended the ESO from continuous space to discrete space but also presented the discrete linear specific form and stability analysis of ESO. Wang and Deng [5] proposed an operatorbased robust nonlinear multivariable tracking control for a manipulator with uncertainties by using a robust right coprime factorization approach and discussed the robust stability in the presence of model uncertainties. Deng and $\mathrm{Bu}[6,7]$ discussed the robust control for nonlinear feedback 
system with unknown perturbations based on the robust right coprime factorization. These research results have been widely used in engineering practice. For example, Hasanzadeh et al. [8] used ESO in the frequency control of the multiregion load and obtained better anti-interference ability than the traditional method. Lin et al. [9] designed a rotor flux observer based on ESO to solve the problem of rotor resistance uncertainty. Shi and Chang [10] designed a timeoptimal control strategy based on ESO and integrated it into an accurate point-to-point movement system. Chan et al. [11] used ESO into two-degree-of-freedom control system of mechanical arms. Compared with a traditional controller, the unique advantages of ESO are embodied in the following aspects. ESO was compared with a sliding mode observer in [12], and the results showed that the upper bound of the interference does not need to be known. However, chattering phenomenon exists in the output of the sliding mode observer. ESO was compared with a high-gain observer in [13] and was used to estimate the unobservable and extended states of the system according to the system input and output. A set of mature experience formulas is available for reference during the parameter setting of ESO. A setting method for ESO parameters was presented in [14].

Research on the posture control of satellite cameras has shown some achievements in recent years. Guan and Zheng [15] introduced an integrated design method of posture control in satellite cameras. Xiubin et al. [16] introduced a control method for the side swing of satellite cameras. However, few reports have focused on the use of ESO in the speed loop control of the pitching axis of satellite cameras.

The adjustment of the pitching posture of satellite camera is presented under the environment of weightlessness. Therefore, a small interference will cause a serious error. Hence, the control system needs a control strategy with stronger antiinterference ability. The control strategy based on ESO does not depend on the precise model of a control object and can estimate the disturbances of a system in real time. This control strategy has a stronger anti-interference ability and is used in this study.

This study analyzed the mathematical models for the actuator permanent magnet synchronous motor (PMSM) of the pitching axis. General-type ESO principles are presented first. The features of ESO nonlinear functions were then analyzed. Given that the disturbances in the speed of satellite cameras need to be eliminated, an ESO was designed and used in the speed loop control of the pitching axis of satellite cameras. The advantages of this type of control strategy were verified by comparing it with a PI control strategy.

\section{Principle and Models}

2.1. Principle of Pitching Axis. The pitching posture of satellite cameras needs adjusting when tracking objects. The pitching axis of satellite cameras can achieve this function. The control schematic diagram of pitching posture of satellite camera is shown in Figure 1. The adjustment of pitching posture is realized on the camera posture rotating platform by using the control board. The PMSM is used as the actuator in the control system of the pitching axis, where $R_{a}$ is the resistance

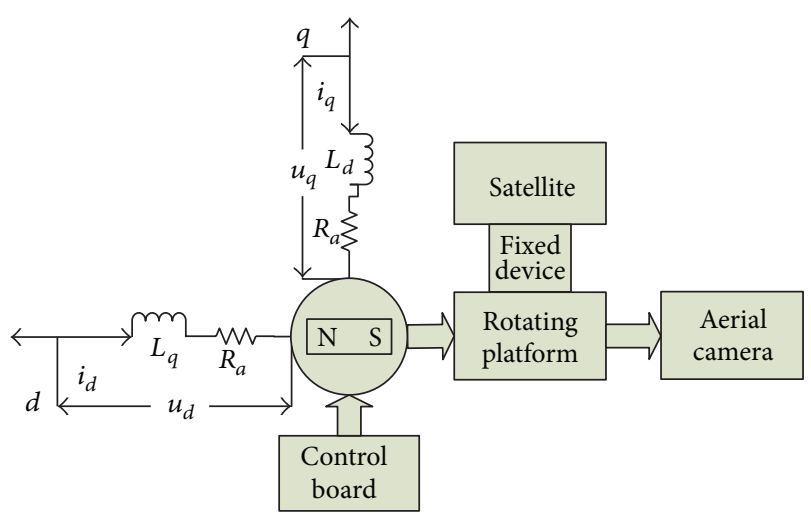

FIGURE 1: The control schematic diagram of pitching posture of satellite camera.

of stator winding. $u_{d}, u_{q}$ are the equivalent voltage of axes $d$ and $q$, respectively. $i_{d}, i_{q}$ are the equivalent current of axes $d$ and $q$, respectively. $L_{d}, L_{q}$ are the equivalent inductance of axes $d$ and $q$, respectively. A three-loop AC servo control system has a current loop, a speed loop, and a position loop used as the control strategy [17]. The speed loop plays an important role in this system. A high-precision speed loop can restrain the uncertain disturbances of the current loop and position loop.

2.2. PMSM Models. The control schematic diagram of pitching posture of satellite camera shows that the control board drives the PMSM. The PMSM drives the rotating platform which was fixed on satellite by fixed device. The rotating platform drives the satellite camera to control the pitching posture. This control function is realized by the control method of the motor speed. Therefore, the models of the actuator PMSM are established in this study. The PMSM models include voltage equation, flux equation, torque equation, and kinematic equation. The following assumptions are presented. First, the saturation effects of iron core are ignored. Second, the air-gap magnetic field shows a normal distribution. Third, the wastage of the eddy current and magnetic field is ignored. Fourth, no damper winding on the motor rotor exists. The kinematic equation of PMSM on the two-phase coordinate system (i.e., the $d q$ coordinate system) is shown as follows:

$$
\dot{\omega}=\frac{1}{J}\left(T_{e}-T_{L}-B \omega\right),
$$

where $B$ is the friction coefficient, $J$ is the rotational inertia of the motor, $\omega$ is the angular speed of the motor rotor, $T_{e}$ is the electromagnetic torque of the motor, and $T_{L}$ is the load torque of the motor.

\section{General Form of ESO}

The classic PI control strategy focuses on the elimination of errors but does not predict and estimate the variable quantities of the system. Hence, this strategy cannot control the magnitude of the integral. The robust performance of 
the controller is greatly improved if the variable quantities of the system can be predicted and estimated in real time. Therefore, a new control strategy based on ESO is presented. The nonlinear dynamics, model uncertainty, and external disturbances of the system are considered as extended states that need to be observed.

A nonlinear single-input single-output system with unknown disturbances is expressed as follows:

$$
x^{(n)}=f\left(x, \ldots, x^{(n-1)}, t\right)+w(t)+b u(t)
$$

where $f\left(x, \ldots, x^{(n-1)}, t\right)$ is an unknown function constituted by the system state variables, $w(t)$ is the unknown external disturbances of the system, $u(t)$ is the input, $x, \ldots, x^{(n-1)}$ are the state variables of the system, and $x(t)$ can be measured.

Assume that $x_{1}=x(t), x_{2}=x^{\prime}(t), \ldots, x_{n}=x^{(n-1)}(t)$. The state space of the system can be expressed as follows:

$$
\begin{gathered}
\dot{x}_{1}=x_{2} \\
\dot{x_{2}}=x_{3} \\
\vdots \\
\dot{x}_{n}=f\left(x_{1}, x_{2}, \ldots, x_{n}\right)+w(t)+b u(t) .
\end{gathered}
$$

Assume that $a(t)=f\left(x, x^{(1)}, \ldots, x^{(n-1)}, t\right)+w(t)$. The ESO of the system can be expressed as follows:

$$
\begin{gathered}
\dot{z}_{1}=z_{2}-g_{1}\left(z_{1}-x(t)\right) \\
\dot{z}_{2}=z_{3}-g_{2}\left(z_{1}-x(t)\right) \\
\vdots \\
\dot{z}_{n}=z_{n+i}-g_{n}\left(z_{1}-x(t)\right)+b u(t) \\
z_{n+1}=-g_{n+1}\left(z_{1}-x(t)\right),
\end{gathered}
$$

where $z_{1} \rightarrow x(t), \ldots, z_{n} \rightarrow x^{n-1}(t), z_{n+1} \rightarrow a(t)$ are the estimations of unknown functions $f\left(x, x^{(1)}, \ldots, x^{(n-1)}, t\right)$ and disturbances $w(t)$. A nonlinear system was constructed by treating $x(t)$ as an input. If nonlinear functions $g_{1}(z), g_{2}(z), \ldots, g_{n+1}(z)$ were selected appropriately, the state variables of the nonlinear system could track the state variables of the original system and $a(t)$. Hence, the ESO of the system can be obtained. Furthermore, the ESO can compensate the uncertain objects and external disturbances in the original system. A nonlinear function is the mathematical fitting of big error and small gain or small error and big gain. This function is generally selected as a nonlinear combination power function and can be shown as follows [18]:

$$
\mathrm{fal}(\varepsilon, \alpha, \delta)=\left\{\begin{array}{ll}
|x|^{\alpha} \operatorname{sign}(x), & |x|>\delta \\
\frac{x}{\delta^{1-\alpha}}, & |x| \leq \delta,
\end{array} \quad \delta>0\right.
$$

Simulations were carried out based on the fal function to evaluate the function of performances. The curve of the fal

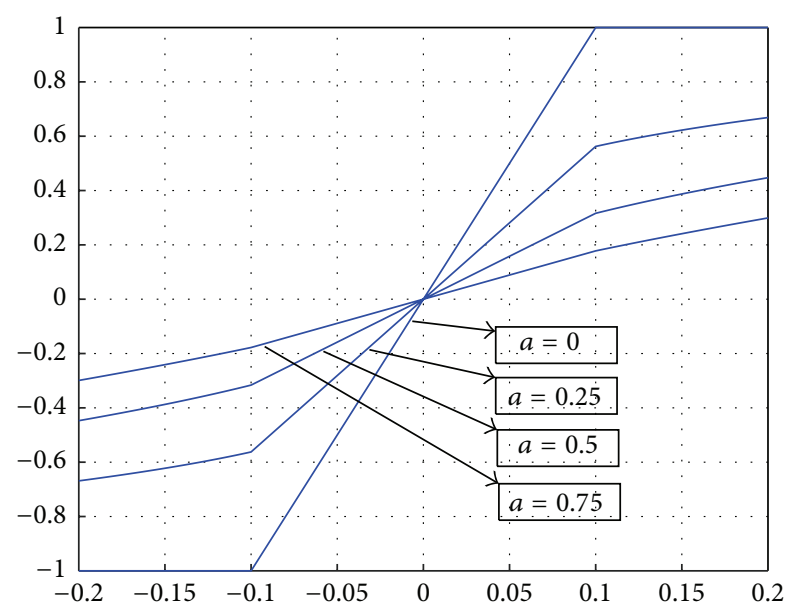

Figure 2: The curve of fal function when $\delta=0.1$.

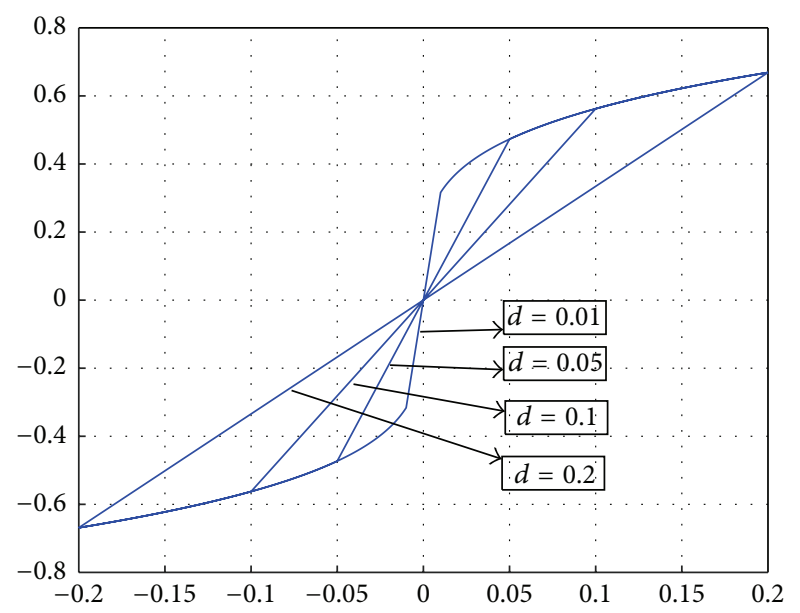

FIgURE 3: The curve of fal function when $\alpha=0.25$.

function when $\delta=0.1$ is shown in Figure 2. The curve of the fal function when $\alpha=0.25$ is shown in Figure 3 .

The simulation results show that the value of $\alpha$ influences the degree of nonlinearity of fal function. According to experience, the value of $\alpha$ is chosen between zero and one. When $\alpha=0$, the degree of nonlinearity of fal function is most serious. When $\alpha=0.75$, the degree of linearity of fal function is best. The value of $\delta$ is the linear interval width of the fal function and is related to the error range of the system. In practical applications, the value of $\delta$ is generally selected as $\delta=0.01$. So when the input is an error signal, the system could achieve the stable status rapidly by adjusting the parameter values of fal function.

\section{Design of Speed Loop ESO}

The design process of ESO is based on the following steps. First, the ESO parameters are designed, and the stability of ESO is ensured. Second, the disturbance observation bandwidth of ESO can be set according to the characteristics of object disturbance. Third, the ESO parameters could be set by using the parameter tuning method. Finally, the output 


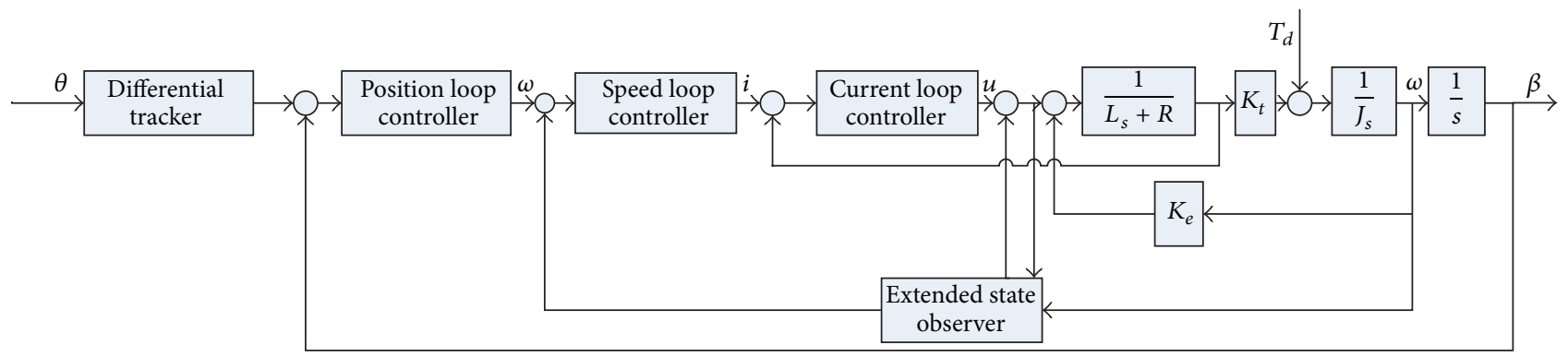

FIGURE 4: Schematic of the control system of pitching axis.

signal of ESO is amended according to the case of the noise interference of object output signal. The schematic of the control system of pitching axis is shown in Figure 4.

\subsection{Design of the Speed Loop ESO for the Pitching Axis of Satel-} lite Cameras. The key of the speed loop control technique is the control of electromagnetic torque. The feedback control strategy of the speed loop can be designed on the basis of the speed error and the given speeds, and the test speeds are obtained. Then effective torque control can be achieved.

The speed loop is also influenced by uncertain disturbances such as the rotational inertia and load torque. Therefore, the main purpose of the speed loop controller is to eliminate these influences. The disturbances and error of model linearization are eliminated by ESO. Hence, the purpose of eliminating uncertain factors can be achieved. Considering the unknown external disturbances, the input is selected as $u=i_{q}$, and the output is selected as $y=\omega$. The PMSM kinematic equation can also be expressed as follows:

$$
\dot{\omega}=\frac{3 p \phi_{f}}{2 J} i_{q}-\frac{B}{J} \omega-\frac{T_{L}}{J} .
$$

The ESO of the speed loop can be designed by the following steps. Some definitions are given as follows:

$$
\begin{aligned}
x & =\omega, \\
a & =-\frac{B}{J}, \\
f(x, t) & =a x, \\
b & =1.5 \frac{p \phi_{f}}{J}, \\
w(t) & =-\frac{T_{L}}{J}, \\
u & =i_{q}, \\
y & =\omega .
\end{aligned}
$$

The expression of kinematic equation with extended states can be shown as follows:

$$
\begin{aligned}
& \dot{x}=f(x, t)+w(t)+b u, \\
& y=x .
\end{aligned}
$$

The ESO of the system is designed as follows:

$$
\begin{aligned}
& \dot{z}_{1}=z_{2}-g_{1} \mathrm{fal}\left(e, \alpha_{1}, \delta_{1}\right)+f_{0}\left(z_{1}\right)+b_{0} u \\
& \dot{z}_{2}=-g_{2} \mathrm{fal}\left(e, \alpha_{2}, \delta_{1}\right)
\end{aligned}
$$

where $z_{1}$ is the tracking of the motor speed signal $\omega, z_{2}$ is the tracking of the motor load torque disturbance $T_{L}$, and $\alpha_{1}$, $\alpha_{2}$ are nonlinear factors and satisfy the condition $0<\alpha_{2}<$ $\alpha_{1} \leq 1$. This study selected the numerical values of $\alpha_{1}, \alpha_{2}$ as $\alpha_{1}=1, \alpha_{2}=0.5$. $\delta_{1}$ is a filtering factor with a numerical value of $\delta_{1}=0.01$. The two expressions include $e=z_{1}-\omega$ and $f_{0}\left(z_{1}\right)=-B z_{1} / J$. Therefore, load torque can be estimated by these methods.

4.2. Stability Analysis of the Speed Loop ESO. The speed loop ESO is selected to perform stability analysis. An uncertain nonlinear single-input single-output system that applies unknown external disturbances is given as follows [19]:

$$
\dot{y}=f+b_{0} u
$$

where $f=g+\left(b-b_{0}\right) u$ is an extended state, a differential function, and the sum of disturbances that need to be estimated. $b$ is the high-frequency gain whose precise numerical value is difficult to obtain. $b_{0}$ is the estimation value of $b$.

Assume that $f=x_{2}, \dot{f}=h$. Then the following equation can be derived:

$$
\begin{aligned}
y & =x_{1}, \\
\dot{x_{1}} & =x_{2}+b_{0} u, \\
\dot{x_{2}} & =h .
\end{aligned}
$$

The above equation can be simplified as follows:

$$
\begin{aligned}
{\left[\begin{array}{l}
\dot{x_{1}} \\
\dot{x_{2}}
\end{array}\right] } & =\left[\begin{array}{ll}
0 & 1 \\
0 & 0
\end{array}\right]\left[\begin{array}{l}
x_{1} \\
x_{2}
\end{array}\right]+\left[\begin{array}{l}
b_{0} \\
0
\end{array}\right] u+\left[\begin{array}{l}
0 \\
1
\end{array}\right] h \\
y & =\left[\begin{array}{ll}
1 & 0
\end{array}\right]\left[\begin{array}{l}
x_{1} \\
x_{2}
\end{array}\right] .
\end{aligned}
$$

The ESO of the above system can be designed as follows:

$$
\begin{aligned}
& \dot{z}_{1}=z_{2}+\beta_{1}\left(y-z_{1}\right)+b_{0} u \\
& \dot{z}_{2}=\beta_{2}\left(y-z_{1}\right)
\end{aligned}
$$


$\beta_{1}, \beta_{2}$ are the parameters of ESO. The state-space expression of the above equation can be shown as follows:

$$
\left[\begin{array}{c}
\dot{z}_{1} \\
\dot{z_{2}}
\end{array}\right]=\left[\begin{array}{cc}
-\beta_{1} & 1 \\
-\beta_{2} & 0
\end{array}\right]\left[\begin{array}{l}
z_{1} \\
z_{2}
\end{array}\right]+\left[\begin{array}{cc}
b_{0} & \beta_{1} \\
0 & \beta_{2}
\end{array}\right]\left[\begin{array}{l}
u \\
y
\end{array}\right] .
$$

The following equation can be obtained by performing Laplace transformation on the above equation:

$$
\begin{aligned}
& z_{1}(s)=\frac{b_{0} s}{s^{2}+\beta_{1} s+\beta_{2}} u(s)+\frac{\beta_{1} s+\beta_{2}}{s^{2}+\beta_{1} s+\beta_{2}} y(s), \\
& z_{2}(s)=\frac{-\beta_{2} b_{0}}{s^{2}+\beta_{1} s+\beta_{2}} u(s)+\frac{\beta_{2} s}{s^{2}+\beta_{1} s+\beta_{2}} y(s) .
\end{aligned}
$$

The state-space expression of the above equation can be shown as follows:

$$
\left[\begin{array}{l}
z_{1}(s) \\
z_{2}(s)
\end{array}\right]=\left[\begin{array}{cc}
\frac{b_{0} s}{s^{2}+\beta_{1} s+\beta_{2}} & \frac{\beta_{1} s+\beta_{2}}{s^{2}+\beta_{1} s+\beta_{2}} \\
\frac{-\beta_{2} b_{0}}{s^{2}+\beta_{1} s+\beta_{2}} & \frac{\beta_{2} s}{s^{2}+\beta_{1} s+\beta_{2}}
\end{array}\right]\left[\begin{array}{l}
u(s) \\
y(s)
\end{array}\right] .
$$

The following equation can be obtained by performing Laplace transformation on formula (10):

$$
s y(s)=f(s)+b_{0} u(s) .
$$

The following equation can be obtained by combining formula (16) with (17):

$$
z_{2}(s)=\frac{\beta_{2}}{s^{2}+\beta_{1} s+\beta_{2}} f(s) .
$$

If input $f(t)$ is a step input, the steady-state error can be calculated as follows:

$$
\begin{aligned}
\lim _{t \rightarrow \infty} e(t) & =\lim _{t \rightarrow \infty}\left[z_{2}(t)-f(t)\right]=\lim _{s \rightarrow 0} s \cdot \frac{1}{s} \cdot e(s) \\
& =\lim _{s \rightarrow 0}\left[\frac{-s\left(s+\beta_{1}\right)}{s^{2}+\beta_{1} s+\beta_{2}}\right]=0 .
\end{aligned}
$$

Therefore, the ESO can achieve robust stability when its state matrix meets the condition of Hurwitz stability.

4.3. Parameter Design of the Speed Loop ESO. To eliminate observation errors, $\beta_{1}$ must meet the condition $\left|\beta_{1}\right| \gg 0$ at the high-frequency stage, and $\beta_{2}$ must meet the condition $\left|\beta_{2}\right| \gg 0$ at a low-frequency stage. However, if $\beta_{1}$ and $\beta_{2}$ are oversized, the sensitivity of ESO to noise will increase and the stability of the closed-loop system will be negatively affected. Therefore, the observation accuracy and sensitivity of ESO to noise should be considered when evaluating parameters $\beta_{1}$ and $\beta_{2}$. The system poles are assigned in the point with a value of $h$. If the case of the above conditions is met and the stability of ESO is guaranteed, parameters $\beta_{1}, \beta_{2}$ and poles $h$ satisfy the following relation [14]:

$$
s^{2}+\beta_{1} s+\beta_{2}=(s+h)^{2} .
$$

Hence, the following equation can be obtained:

$$
\begin{aligned}
& \beta_{1}=2 h, \\
& \beta_{2}=h^{2} .
\end{aligned}
$$

Therefore, only two ESO parameters, namely, $\beta_{1}, b_{0}$, need to be adjusted. A large number of simulation experiments give the following rules during the adjustment of the two parameters.

On one hand, a larger $\beta_{1}$ leads to the faster observation and control compensation of errors by ESO. However, the sensitivity of ESO to noise is increased and the sample frequency of the system becomes restricted. During the adjustment of $\beta_{1}$, the numerical value of $\beta_{1}$ should gradually increase until it meets the performance requirements.

On the other hand, the numerical value of $b_{0}$ should be close to the actual high-frequency gain of the system as possible. According to the initial-value theorem, the numerical value can be determined by calculating the system's rising rate of the initial time for step response. If the numerical value of $b_{0}$ is difficult to calculate, it can be debugged from big to small. A stronger control action corresponds to a smaller stability margin.

The numerical values of $\beta_{1}, b_{0}$ can be chosen as $\beta_{1}=$ $1, b_{0}=1$. If the output of ESO appears to vibrate, the numerical value of $\beta_{1}$ must be decreased until the output of ESO appears smooth. Otherwise, the numerical value of $\beta_{1}$ should be increased until the output of ESO appears to vibrate. If the adjustment of $\beta_{1}$ cannot meet the requirements, the numerical value of $b_{0}$ should be adjusted to achieve good ESO performance with good estimated accuracy on the premise of low noise sensitivity.

By considering the assigned system poles on the position of -10 , the numerical values of $\beta_{1}$ and $\beta_{2}$ can be calculated as $\beta_{1}=10$ and $\beta_{2}=25$. The numerical value of $b_{0}$ is selected as $b_{0}=1$, and the ESO of the system is designed.

4.4. Disturbance Compensation. The input of the system can be compensated if ESO can estimate the state variables of the system in real time. The system can approximately simplify an integral series connection system after compensation. Therefore, the nonlinear state error feedback control strategy can use a linear proportional function instead of a nonlinear function. The disturbance outputs are observed by ESO to control the input. Then a controlling regularity can be designed as follows:

$$
u=\frac{u_{0}-z_{2}}{b_{0}}
$$

By combining formula (10) with (22), the following equation can be obtained:

$$
\dot{y}=f+b_{0}\left(\frac{u_{0}-\tilde{f}}{b_{0}}\right)=u_{0} .
$$

The open-loop system can be approximated into an integration element. The pole assignment can be realized by a proportionality coefficient:

$$
u_{0}=k_{p}\left(r-z_{1}\right) \text {. }
$$




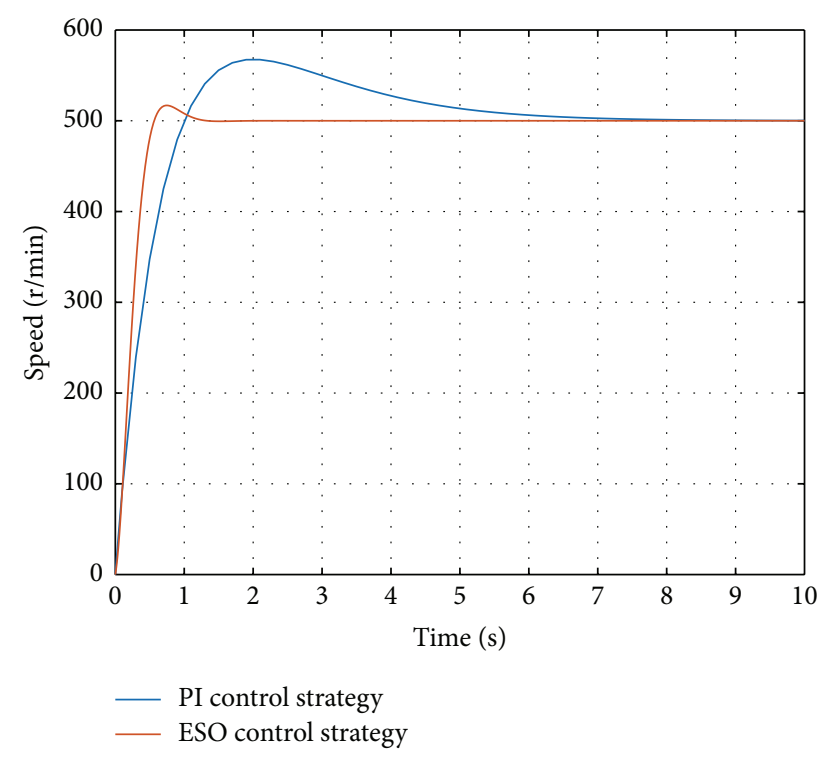

FIGURE 5: Motor speed response curve of nonload starting.

Therefore, the expected dynamic characteristics of the closedloop system can be expressed as follows:

$$
G_{c}(s)=\frac{y(s)}{r(s)}=\frac{1}{s+k_{p}} .
$$

\section{Simulation Experiments}

The control strategy is taken to compare with the PI control strategy to accurately judge the performances of the control strategy based on ESO. A PMSM produced by Kollmorgen (KBM-43X01) was selected as the executing agency of the system. Table 1 shows the motor parameters.

Simulations were carried out in the following four cases for two types of control strategies.

First, nonload starting. A given speed of $500 \mathrm{r} / \mathrm{min}$ is suddenly applied on the rotor for motor nonload starting at the time $0 \mathrm{~s}$. Figure 5 shows the motor speed response curve under the two types of control strategies. This figure shows that the control strategy based on ESO has a shorter adjusting time and smaller overshoot amount than the PI control strategy. From the simulation results it can be seen that the overshoot amount of the control strategy based on ESO is $10.098 \%$ less than that of the PI control strategy and the response time of the control strategy based on ESO is $5.885 \mathrm{~s}$ less than that of the PI control strategy. The speed loop parameters of the overshoot amount and the response time for the PI control strategy and ESO control strategy for nonload starting are shown in Table 2.

Second, the external load disturbance is applying on the rotor suddenly. The initial motor speed is given as $500 \mathrm{r} / \mathrm{min}$. An external load disturbance of $300 \mathrm{r} / \mathrm{min}$ was suddenly applied on the rotor at the time $5 \mathrm{~s}$. Figure 6 shows the motor speed response curve under the two types of control strategies. From the simulation results it can be seen that after the external load disturbance applied on the rotor suddenly,

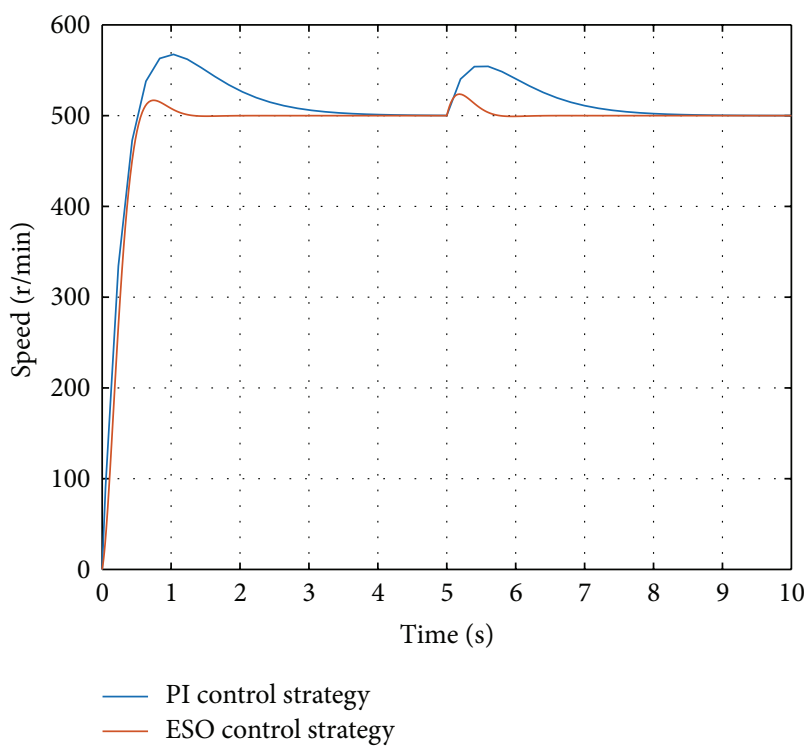

FIGURE 6: Motor speed response curve of external load disturbance suddenly applied.

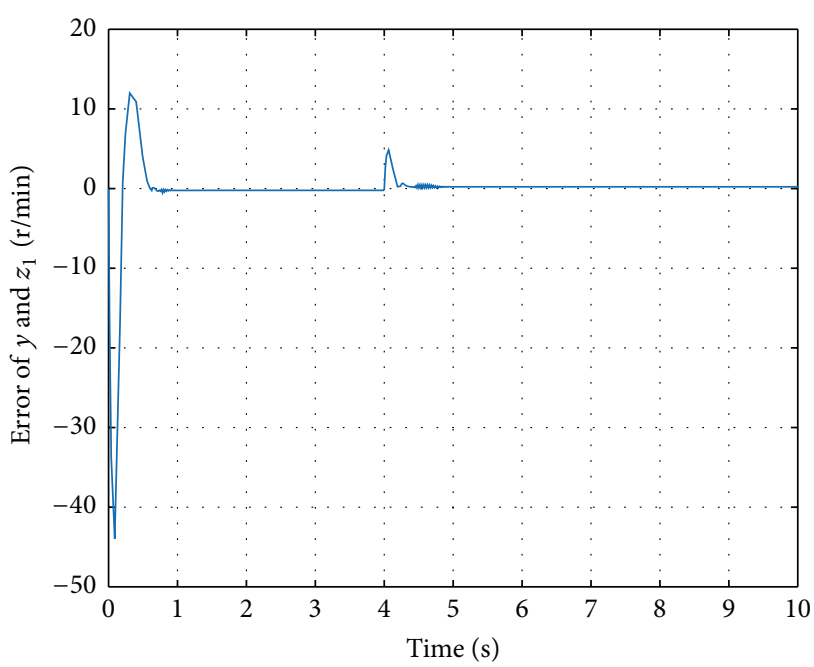

FIGURE 7: Error of $y$ and $z_{1}$.

the control strategy based on ESO has a smaller response curve fluctuation, shorter recovery time, and smaller load disturbance influence than that of the PI control strategy. The simulation results show that the overshoot amount of the control strategy based on ESO is $6.068 \%$ less than that of the PI control strategy and the response time of the control strategy based on ESO is 2.341 s less than that of the PI control strategy. The speed loop parameters of the overshoot amount and recovery time for the PI control strategy and ESO control strategy on the case of the external load disturbance applying on the rotor suddenly are shown in Table 3 .

Simulation results of the error of $y$ and $z_{1}$ are shown in Figure 7 and the errors of $d y / d t$ and $z_{2}$ are shown in Figure 8.

The simulation results show that the compensation time of disturbance is about $0.673 \mathrm{~s}$ when the bandwidth of 
TABLE 1: Parameters of the experiment motor.

\begin{tabular}{|c|c|c|c|c|c|c|c|c|c|c|}
\hline \multirow[t]{2}{*}{ Type } & $\begin{array}{l}\text { Power } \\
\text { supply } \\
\text { voltage }\end{array}$ & $\begin{array}{l}\text { Rated } \\
\text { power }\end{array}$ & $\begin{array}{c}\text { Maximum } \\
\text { nonload } \\
\text { speed }\end{array}$ & $\begin{array}{l}\text { Continuous } \\
\text { current }\end{array}$ & $\begin{array}{c}\text { Continuous } \\
\text { torque }\end{array}$ & $\begin{array}{c}\text { Peak } \\
\text { current }\end{array}$ & $\begin{array}{l}\text { Peak } \\
\text { torque }\end{array}$ & $\begin{array}{l}\text { Motor pole } \\
\text { logarithmic }\end{array}$ & Resistance & Inductance \\
\hline & VAC & $\mathrm{W}$ & $\mathrm{r} / \mathrm{min}$ & A & $\mathrm{N} \cdot \mathrm{m}$ & A & $\mathrm{N} \cdot \mathrm{m}$ & & $\Omega$ & $\mathrm{mH}$ \\
\hline KBM-43X01 & 400 & 2400 & 2750 & 5.1 & 6.11 & 18 & 18 & 16 & 2.9 & 6.8 \\
\hline
\end{tabular}

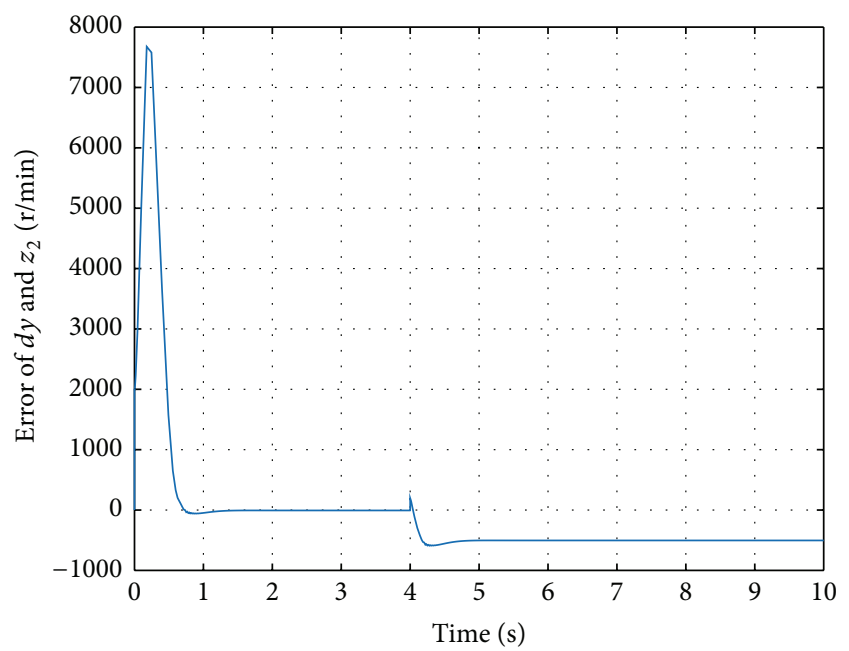

FIGURE 8: Error of $d y / d t$ and $z_{2}$.

TABLE 2: Response parameters of the system for nonload starting.

\begin{tabular}{lcc}
\hline Strategy & Overshoot amount & Response time \\
\hline PI & $13.461 \%$ & $7.098 \mathrm{~s}$ \\
ESO & $3.363 \%$ & $1.213 \mathrm{~s}$ \\
\hline
\end{tabular}

TABLE 3: Response parameters of external load disturbance applied on rotor suddenly.

\begin{tabular}{lcc}
\hline Strategy & Overshoot amount & Response time \\
\hline PI & $10.813 \%$ & $2.973 \mathrm{~s}$ \\
ESO & $4.745 \%$ & $0.632 \mathrm{~s}$ \\
\hline
\end{tabular}

regulating error is $5 \%$. That is to say, the state variables $z_{1}$ and $z_{2}$ can indicate $y$ and $d y / d t$ timely, respectively. Therefore the control strategy based on ESO can eliminate the influence of the disturbance rapidly and can achieve a result that the steady-state error is zero.

Third, in the case of the speed of motor changing suddenly, the initial motor speed is $500 \mathrm{r} / \mathrm{min}$. The motor speed decreases from $500 \mathrm{r} / \mathrm{min}$ to $200 \mathrm{r} / \mathrm{min}$ at the time $4 \mathrm{~s}$. The motor speed response curve under the two types of control strategies is shown in Figure 9. The figure shows that the control strategy based on ESO has smaller response curve fluctuations and better recovery capability than the PI control strategy when the speed of motor changes suddenly. From the simulation results it can be seen that the overshoot amount of the control strategy based on ESO is 3.034\% less than that of the PI control strategy and the response time of the control strategy based on ESO is 2.688 s less than that of the PI
TABLE 4: System response parameters when the speed of motor changes suddenly.

\begin{tabular}{lcc}
\hline Strategy & Overshoot amount & Response time \\
\hline PI & $5.055 \%$ & $3.367 \mathrm{~s}$ \\
ESO & $2.021 \%$ & $0.679 \mathrm{~s}$ \\
\hline
\end{tabular}

TABLE 5: Parameters of motor speed error.

\begin{tabular}{lcc}
\hline Strategy & Speed maximum error & Error vanishing time \\
\hline PI & $69.797 \mathrm{r} / \mathrm{min}$ & $7.596 \mathrm{~s}$ \\
ESO & $19.703 \mathrm{r} / \mathrm{min}$ & $1.433 \mathrm{~s}$ \\
\hline
\end{tabular}

control strategy. The speed loop parameters of the overshoot amount and recovery time for the PI control strategy and ESO control strategy when the speed of motor changes suddenly are shown in Table 4.

Fourth, response analysis is conducted on the motor speed error. The speed error response curve can be obtained in Figure 10 by comparing the actual motor speed output with the given speed. The figure shows that the speed error of the control strategy based on ESO decreases to $0 \mathrm{r} / \mathrm{min}$ faster than that of the PI control strategy. From the simulation results it can be seen that the speed maximum error of the control strategy based on ESO is $50.094 \mathrm{r} / \mathrm{min}$ less than that of the PI control strategy and the error vanishing time of the control strategy based on ESO is 6.163 s less than that of the PI control strategy. The parameters of motor speed error are shown in Table 5 .

\section{Conclusions}

A speed control system for the pitching axis of satellite cameras based on an ESO was designed. The unknown states and uncertain factors of the system were estimated by the ESO. This control strategy compensates the influences produced by disturbances, and some good control performances are obtained. The simulation results show that the control strategy based on ESO has better control effects than the PI control strategy. This research provides a theoretical basis for future research. The application of ESO to the drift axis control system of satellite cameras, as well as the study of high-order ESO, will be conducted in future works.

\section{Competing Interests}

The authors declare that they have no competing interests. 


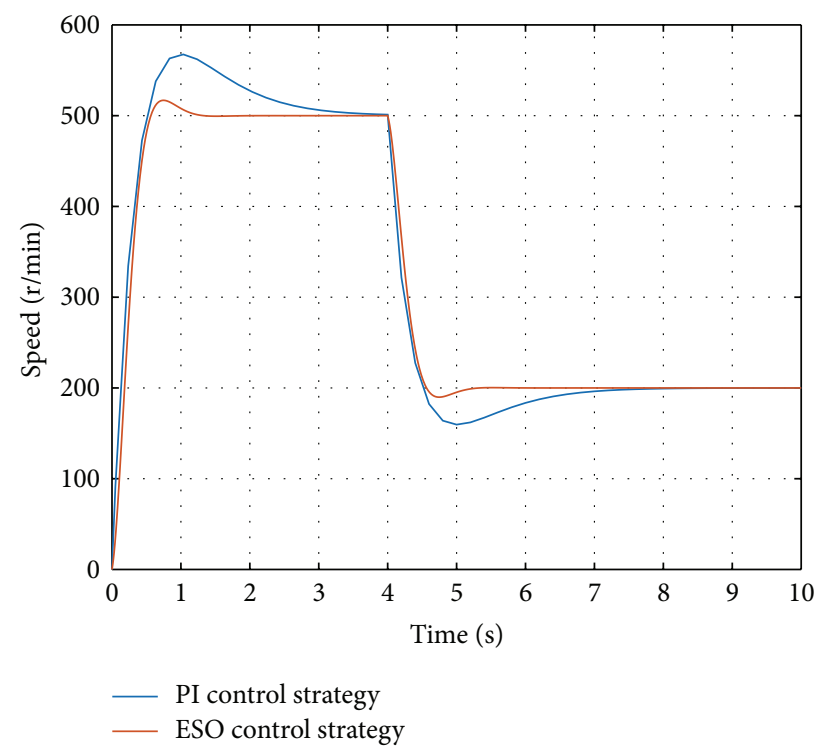

FIGURE 9: Motor speed response curve when the speed of motor changes suddenly.

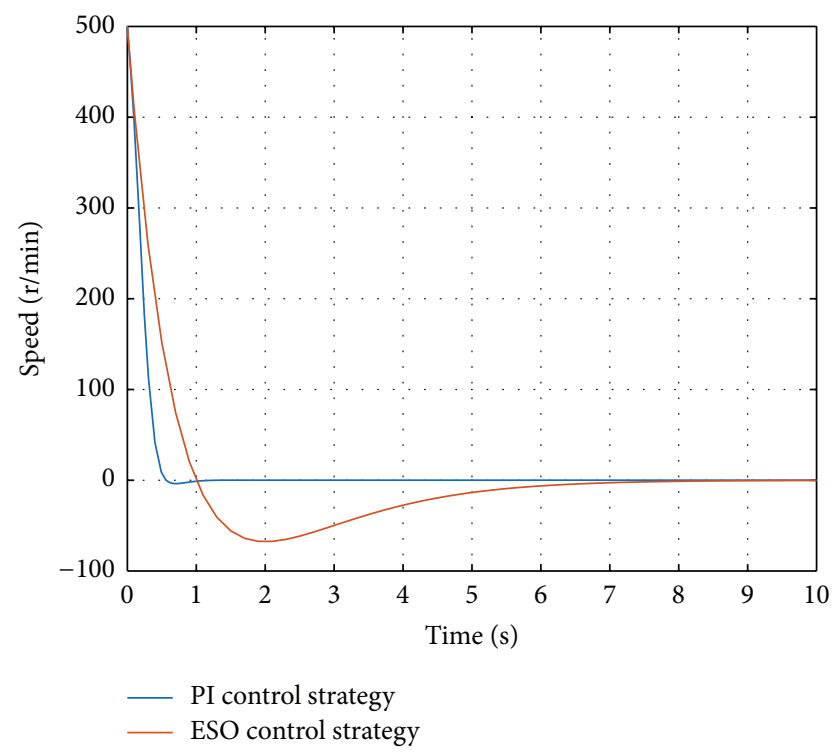

FIGURE 10: Motor speed error response curve.

\section{Acknowledgments}

This work was supported by the Key Project of Natural Science by Education Department of Anhui Province (no. KJ2015A316), the Major Project of Natural Science by Education Department of Anhui Province (no. KJ2014ZD004), Outstanding Young Talents at Home Visit the School Training Project (no. gxfxZD2016101), Natural Science Foundation of Anhui Province of China (no. 1508085QE83), and the Key Science and Technologies Program of Anhui Province (no. 1301021005).

\section{References}

[1] J.-Q. Han, Active Disturbance Rejection Control Technique, National Defense Industry Press, Beijing, China, 2008.

[2] X.-X. Yang and Y. Huang, "Capabilities of extended state observer for estimating uncertainties," in Proceedings of the American Control Conference (ACC '09), pp. 3700-3705, IEEE, St. Louis, Mo, USA, June 2009.

[3] Y.-H. Wang, Y. Yao, and K.-M. Ma, "Error estimation of second order extended state observer," Journal of Jilin University (Engineering and Technology Edition), vol. 40, no. 1, pp. 143-147, 2010.

[4] L.-W. Shao, X.-Z. Liao, Y.-Q. Xia et al., "Stability analysis and synthesis of third order discrete extended state observer," Information and Control, vol. 37, no. 2, pp. 135-139, 2008.

[5] A. Wang and M. Deng, "Robust nonlinear multivariable tracking control design to a manipulator with unknown uncertainties using operator-based robust right coprime factorization," Transactions of the Institute of Measurement and Control, vol. 35, no. 6, pp. 788-797, 2013.

[6] M. Deng and N. Bu, "Robust control for nonlinear systems with unknown perturbations using simplified robust right coprime factorisation," International Journal of Control, vol. 85, no. 7, pp. 842-850, 2012.

[7] M. Deng and N. Bu, "Robust control for nonlinear systems using passivity-based robust right coprime factorization," IEEE Transactions on Automatic Control, vol. 57, no. 10, pp. 25992604, 2012.

[8] M. Hasanzadeh, M. R. Jahed-Motlagh, and M. H. Kazemi, "Decentralized disturbance rejection control of multi-area power systems using generalized extended state observer," in Proceedings of the 20th Iranian Conference on Electrical Engineering (ICEE '12), pp. 940-945, IEEE, Tehran, Iran, May 2012.

[9] F. Lin, C.-P. Zhang, W.-C. Song, L.-W. Jiao, and S.-S. Chen, "Flux observer of induction motor based on extended state observer," Proceedings of the Chinese Society for Electrical Engineering, vol. 23, no. 4, pp. 145-147, 2003.

[10] X. Shi and S. Chang, "Extended state observer-based timeoptimal control for fast and precise point-to-point motions driven by a novel electromagnetic linear actuator," Mechatronics, vol. 23, no. 4, pp. 445-451, 2013.

[11] L. Chan, F. Naghdy, and D. Stirling, "Extended active observer for force estimation and disturbance rejection of robotic manipulators," Robotics and Autonomous Systems, vol. 61, no. 12, pp. 1277-1287, 2013.

[12] Y.-S. Lu, "Sliding-mode disturbance observer with switchinggain adaptation and its application to optical disk drives," IEEE Transactions on Industrial Electronics, vol. 56, no. 9, pp. 37433750, 2009.

[13] H. K. Khalil and L. Praly, "High-gain observers in nonlinear feedback control," International Journal of Robust and Nonlinear Control, vol. 24, no. 6, pp. 993-1015, 2014.

[14] Y.-Q. Zhu, J.-Y. Dong, S.-H. Chen, F.-P. Pan, and D.-H. Li, "Extended state observer based control system design for boilerturbine unit," Journal of the University of Electronic Science and Technology of China, vol. 43, no. 5, pp. 700-705, 2014.

[15] X. Guan and G.-T. Zheng, "Integrated design of space telescope vibration isolation and attitude control," Journal of Astronautics, vol. 34, no. 2, pp. 214-221, 2013.

[16] Y. Xiubin, J. Li, and J. Guang, "Image matching analysis and experiment of spaceborne camera swayed in the end of orbit 
life," Chinese Journal of Space Science, vol. 33, no. 3, pp. 337-345, 2013.

[17] K. Wang, M.-Z. Luo, K. Li, Y. Cao, and Q.-J. Zhang, "Control algorithm optimization of permanent magnet synchronous motor based on state constraints," Electric Machines and Control, vol. 19, no. 2, pp. 33-38, 2015.

[18] J.-F. Mao, A.-H. Wu, G.-Q. Wu, X.-D. Zhang, and G.-X. Wu, "Adaptive sliding mode MPPT control for PMSG-based wind energy conversion systems based on extended state observer," Power System Protection and Control, vol. 42, no. 18, pp. 58-65, 2014.

[19] L. Sun, J. Dong, D. Li, and Y. Xue, "Cascaded load-frequency digital electric-hydraulic regulation of turbine via extended state observer," Proceedings of the Chinese Society of Electrical Engineering, vol. 35, no. 7, pp. 1697-1703, 2015. 


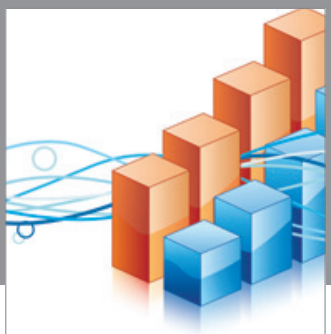

Advances in

Operations Research

vatem alat4

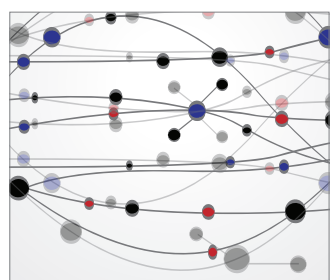

\section{The Scientific} World Journal
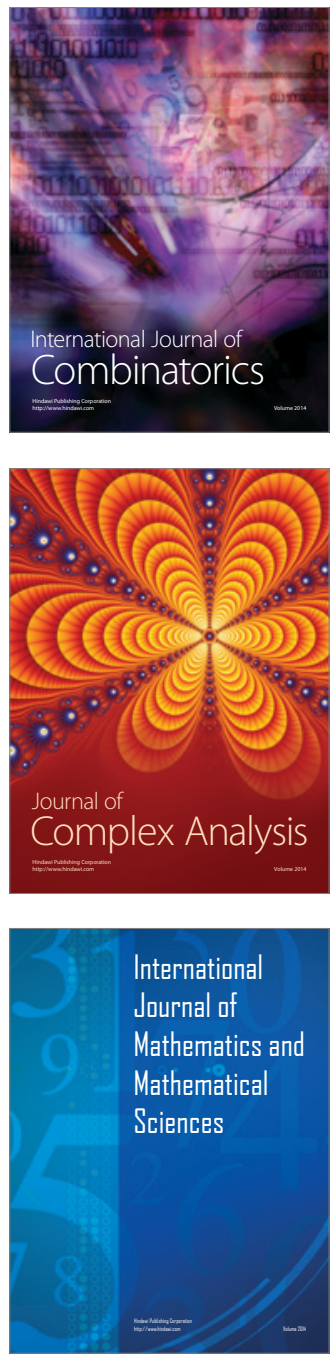
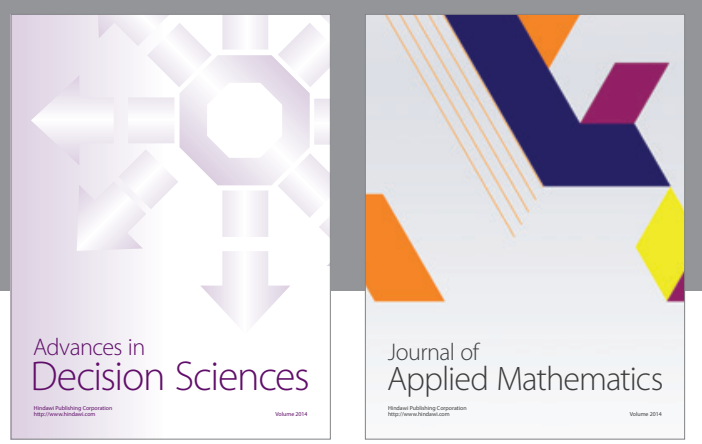

Algebra

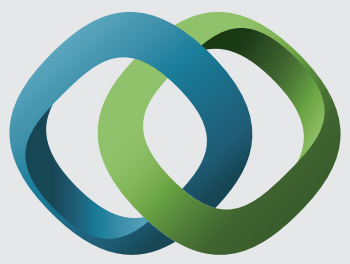

\section{Hindawi}

Submit your manuscripts at

http://www.hindawi.com
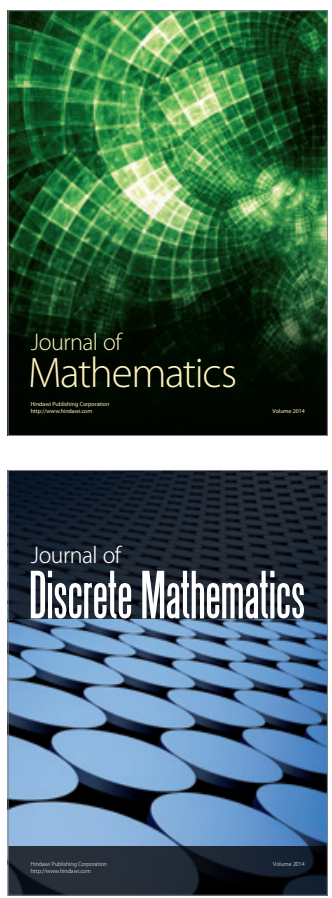

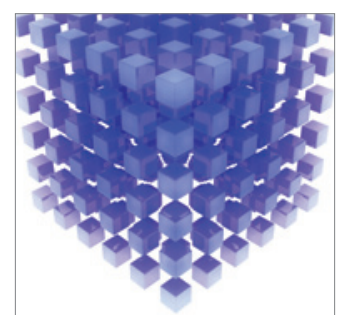

Mathematical Problems in Engineering
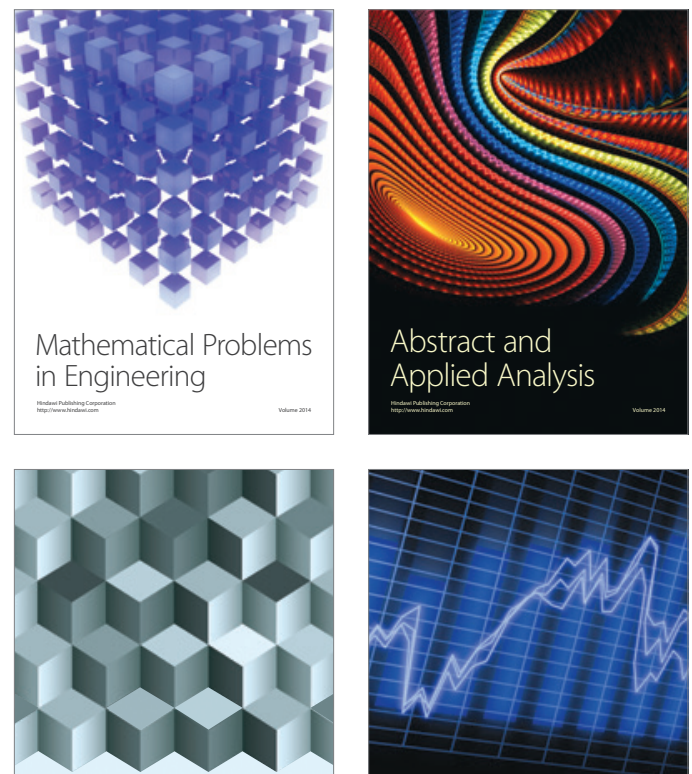

Journal of

Function Spaces

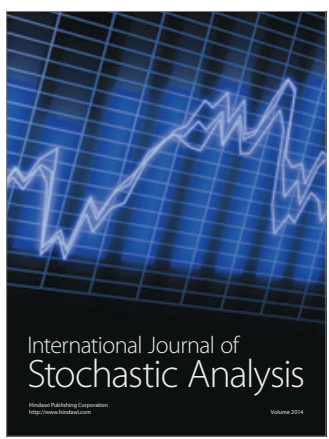

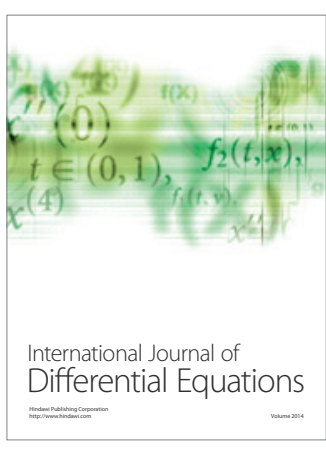
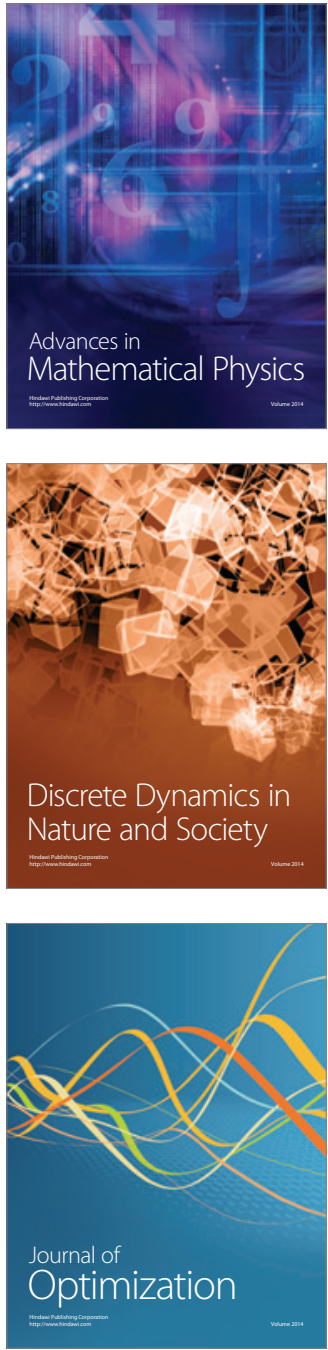